\title{
IDENTIDAD Y DESPLAZAMIENTO LINGÜÍSTICO EN LA COMUNIDAD DE WASAKÍN, MUNICIPIO DE ROSITA, R.A.A.N.
}

Eloy Frank Gomez ${ }^{[1]}$

\section{Resumen}

Se inquirieron los cambios identitarios y lingüísticos de la lengua thuahka al miskitu en Wasakin, una de las diez variantes lingüísticas que antes existían en "la gran familia sumu" ${ }^{[2]}$, fenómeno sociolingüístico de realce en la modernización y los procesos globalizantes.

El pueblo de Wasakín desciende de los sumu-mayagnas, está anclado en el municipio de Rosita, Región Autónoma Atlántico Norte, RAAN. El método de abordaje fueron las entrevistas semi-estructuradas, período del 2004 al 2006. Se apuntaló el uso de un 'mapa de movilidad', instrumento referencial en la investigación sociolingüística de la Academia Británica ${ }^{[3]}$, a través del cual se registraron los puentes comunicativos que se suponen en contextos multilingües.

Entre los resultados se describe la identidad y los cambios culturales, en especial el sociolingüístico y la actitud en los hablantes tuahka, acerca del fenómeno de desplazamiento de la lengua, y cómo se percibe en relación con la identidad cultural de Wasakín.

Se concluye que por las diversas presiones políticas-sociales y la desvalorización de la lengua tuahka frente al miskitu y al panamahka, tal cual lo demuestra la jerarquía etnolingüística de la Costa Caribe, el rescate y revitalización de la lengua tuahka aunque es remota, implica afrontar profundos retos interculturales que se le plantean al Sistema Educativo Autonómico Regional (SEAR).

Palabras claves: identidad cultural, diglosia, poliglosia, contextos multilingües, jerarquía lingüística,

[1] MSc. en Antropología Social con Mención en Desarrollo Humano. asangrarah@yahoo.com.mx

[2] Denominación dada por el padre Smutko (1996).

[3] Este proyecto, en el cual colaboré con la Dra. Jane Freeland, adaptó a la investigación sociolingüística una versión de una técnica de mapeo que tiene su origen en la Investigación Participativa Rural (Participatory Rural Appraisal, PRA). Posteriormente ha sido adaptada como técnica de alfabetización Freiriana (ver Archer y Cottinham 1996). 


\section{Introducción}

La presente narrativa es un tríptico de valoraciones sociolingüísticas desde la perspectiva antropológica, se enfocan las repercusiones del cambio del código lingüístico en la identidad sumu mayangna; los diversos usos de la lengua tuahka y el miskitu; asimismo, la percepción de los comunitarios sumu tuahka de Wasakín, respecto al fenómeno del desplazamiento de la lengua.

En la primera parte se aborda el marco teórico y se reflexiona acerca de la identidad étnica, debate contemporáneo en la antropología y la sociolingüística, en relación con el desplazamiento lingüístico. Esta reflexión parte del preguntar antropológico ¿De qué manera el desplazamiento de la lengua incide en la identidad de un determinado grupo?

El asunto se aborda desde las identidades individuales y las relaciones sociales que se producen con los grupos existentes en la Costa Caribe. Se discute la relación entre lengua e identidad y los escenarios del multilingüismo en los cambios de códigos, específicamente en los fenómenos de diglosia y poliglosia, en la comprensión de que en estos contextos las lenguas ocupan diversas posiciones jerárquicas.

La segunda parte es el habérselas entre los resultados y la discusión, relación dialéctica que deviene de la vivencia cultural en la comunidad. Entrarle a la actualidad de Wasakín es valorar el patrimonio cultural sumu-mayangna; del mismo modo, las referencias mediatas e inmediatas de las investigaciones etnográficas realizadas por sociólogos, antropólogos y lingüistas.

En la tercera parte se esboza etnográficamente la comunidad de Wasakín, en una aproximación al conocimiento de los procesos de contacto y cambios sociales; la estructura de poder tradicional, las características de la población, la situación territorial, costumbres y tradiciones, período 2004-2006. Particular miramiento constituye las relaciones matrimoniales de parejas mixtas entre sumu mayangna y miskitus y, el uso de la lengua en los hogares para conocer el impacto en cuanto al uso de la lengua miskitu.

Además, se retoman los conceptos de la diglosia y poliglosia en relación a las consecuencias de las desiguales políticas sociales, por las cuales las diversas lenguas tienen sus diferentes espacios de uso, dentro y fuera de la comunidad.

Finalmente, se hace una valoración con relación a la percepción y el rol de las madres y padres de familia, al quehacer de las escuelas en cuanto al rescate de la lengua sumu tuahka a través del Programa de Educación Intercultural Bilingüe (PEBI) en la comunidad de Wasakín. 


\section{Revisión de Literatura}

En los últimos 50 años, el tema de la "identidad étnica" tiene actualidad, tanto en la antropología como en la sociolingüística. (Ver, p. ej. Wade, 2000; Modood, 2002, Gómez Suárez 2003).

Del mismo modo, se contextualiza el debate sobre la relación entre lengua e identidad étnica (Ver, p. ej. Jamieson 2001, Hale y Benedicto 2004), en la explicitación de algunos conceptos sociolingüísticos.

Se ha utilizado el concepto de la identidad 'constructivista', por dos razones: La primera con el fin de analizar las realidades de identidad que se producen en la Costa Caribe nicaragüense, a raíz de las relaciones y contactos no sólo con los europeos, sino también en la anterioridad con otros grupos étnicos, sobre todo en el caso de los sumu-mayangna y tuahka de Wasakín. Además, el concepto constructivista tiene cierto relieve en la discusión antropológica, sociológica y sociolingüística (aunque se ha empezado a criticar. Ver, p.ej. Modood, 2000).

La posición constructivista es aquella en la cual las identidades étnicas no son simplemente 'dadas', ni son estáticas o atemporales, sino que cambian más allá de los presupuestos esencialistas. Por lo tanto, en los procesos socioculturales se generan las identidades de fusión, y su estilo de vida refleja una variedad de herencias culturales (Caglar, 1997, en Modood, 2000:175).

Esta perspectiva del cambio sobre la identidad étnica es apropiada a las realidades de la Costa Caribe nicaragüense, porque durante siglos sus habitantes han sido sujetos a cambios causados por el contacto inter-étnico, sea la intervención externa o sea el contacto inter-étnico en una región donde han coexistido muchos grupos (ver p. ej. Von Houwald, 2003 [1990], Jamieson 2001),

\section{Materiales y Métodos}

Entre los métodos, figuran las entrevistas semi-estructuradas con grupos focales de mujeres, jóvenes y personas de la tercera edad. Se apuntaló el uso de un 'mapa de movilidad', instrumento referencial en la investigación sociolingüística de la Academia Británica ${ }^{[4]}$, a través del cual se registraron los puentes comunicativos que se suponen en contextos multilingües.

[4] Este proyecto, en el cual colaboré con la Dra. Jane Freeland, adaptó a la investigación sociolingüística una versión de una técnica de mapeo que tiene su origen en la investigación participativa rural (Participatory Rural Appraisal, PRA). Posteriormente ha sido adaptada como técnica de alfabetización Freiriana (ver Archer y Cottinham 1996). 


\section{Resultados y Discusión}

El triunfo de la Revolución Popular Sandinista para los sumu mayangna concreta los espacios de protagonismo en los subsistemas de educación, y fue a su vez, cuando se conoce la autoreferencia o apropiación para sí, en cuanto a la historia del pueblo sumu mayagna.

Los primeros estudios antropológicos y etnográficos fueron realizados por Lehmann (1910, 1920), y Conzemius (1932), e incluían posiblemente los primeros estudios lingüísticos del sumu. Estos exponen la presencia y el contacto de los diferentes subgrupos que conformaron la familia sumu mayangna. Posteriormente Von Houwald (1984, 2001), cuando era embajador alemán en Nicaragua, dedicó parte de su vida al estudio de los sumu-mayangna, acerca de las tradiciones culturales y la etnohistoria.

Se puede afirmar que actualmente existen reductos de la diglosia en Wasakín y el centro urbano de Rosita; el español es el lenguaje que se utiliza para todas las gestiones en las instituciones del Estado, por ser la lengua franca y oficial de Nicaragua, aunque en el marco de la Autonomía en la Ley de Lenguas (1993) estipula el uso de las lenguas indígenas y étnicas en estos contextos.

La gráfica No. 1 ilustra la jerarquía actual entre las lenguas de la comunidad. Esta jerarquía genera no sólo relaciones de dominio entre el miskitu y el sumu, sino hasta entre las dos variantes del sumu: panamahka y tuahka. En el apartado que trata el trasfondo, se detallará cómo se produjo esa jerarquía lingüística.

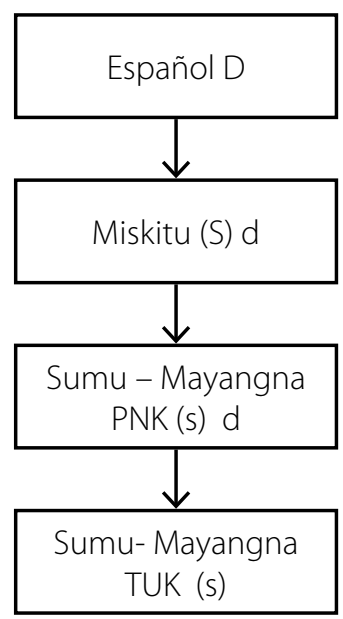

Gráfica No. 1: Jerarquía etnolingüística de la Costa Caribe de Nicaragua 


\section{REVITALIZACIÓN LINGÜÍSTICA Y CULTURAL}

Las notaciones correspondientes a letras mayúsculas indican dominancia (D) o subordinación (S), según percepciones nacionales. Las letras minúsculas indican el sistema local y mucho más complejo de dominancia (d) y subordinación (s).

Se podría afirmar que una situación de diglosia limita el uso de la lengua para expresar la identidad, porque las opciones no son libres, sino que son predeterminadas por las normas sociolingüísticas de diglosia o poliglosia.

Aunque los conceptos de diglosia y poliglosia son útiles para captar las desigualdades de poder entre las lenguas de la comunidad, son demasiado rígidos para captar la complejidad de los comportamientos lingüísticos de Wasakín.

Por eso es pertinente recurrir al concepto de la alternancia de códigos: la tendencia a cambiar libremente, a veces en la misma oración, de una lengua a otra. En Wasakín, por ejemplo, la gente cambia de lengua para ajustarse a las necesidades de otra persona que no habla su lengua.

También hay casos de jóvenes que han aprendido el tuahka como segunda lengua y que no la dominan bien. En este caso, el cambio de código suple una competencia incompleta. A veces, la gente se siente más capaz de expresar ciertas ideas, o hablar de ciertos tópicos en miskitu, en vez de tuahka, posiblemente porque el miskitu ejerce dominio.

\section{Etnografía del trasfondo histórico}

Se sabe que el grupo sumu, ahora mayangna, habitaron la zona de los lagos y poco a poco se extendieron hacia las zonas montañosas y hacia los grandes ríos. Así vivieron hasta la llegada de los conquistadores, cuando se convirtieron en los indios indómitos (Houwald 1984), poblando los territorios que no pudieron ser dominados a la fuerza. Prueba de esta extensión de los sumu parecen ser los topónimos en la lengua sumu que aún permanecen, así como otros que desaparecieron o fueron mutilados por la cartografía moderna que descartaba los nombres indígenas, y la ortografía española que los distorsionaba.

Según observadores en los años 1620, por ejemplo, los que ahora se denominan sumu-mayangnas formaban diferentes grupos, tales como: ulwas, yuskus, kukras, panamahka, tuahka, tawahka, tungla, prinzu, silam, y bawihka (Lehmann, 1932: 16, en Von Houwald 2001: 179). Muchos de estos, por diversas causas desaparecieron con sus lenguas. La explicación más conocida es que la desaparición o decadencia de estos grupos se debió a las incursiones de los miskitus, en alianza con los ingleses, en el siglo XVIII, forzaron a las comunidades sumus a refugiarse hacia las cabeceras de los ríos, abandonando sus territorios originales. Sin embargo, como Jamieson plantea, esto no explica la reducción de la población sumu-mayangna en los siglos XIX y XX. 
El sugiere que "muchas comunidades sumus desaparecieron (...) debido al cambio de idioma al convertirse sus miembros en hablantes del mískitu o del español. Este proceso continúa en el siglo presente." (Jamieson 2001: 7, ver también von Houwald). Por ejemplo, en una asamblea organizada a raíz de la investigación piloto de la British Academy, una anciana de Dibahil contó haber nacido de padres bawihka en una comunidad sumu en el río Kukalaya, los últimos descendientes del grupo bawihka.

Jamieson acentúa, estas comunidades son clasificadas por foráneos como 'mískitas' o 'mestizos' por la lengua que hablan, pero realmente siguen identificándose a sí mismos, y comportándose en muchos otros aspectos como sumus.

La historia oral del pueblo sumu tuahka tiene sus antecedentes desde el año 180o, cuando poblaron diferentes zonas del país y de la región del Atlántico, Lehmann, basándose en esta historia, menciona otras de las regiones asentadas por los tuahkas, donde antiguamente vivían cuando él los estudiaba. Estas regiones son los ríos grandes de Matagalpa, Kukalaya, Bambana, tungkih, Lisawe, Pìspìs, Waspuk y Lakus.

La comunidad sumu tuahka de Wasakín históricamente ha vivido procesos de contacto con otros grupos étnicos, en específico los miskitu, esto ha originado la fusión entre la cultura.

En la actualidad sólo sobreviven cuatro de estos grupos, y sus lenguas también en mayor o menor grado. Los panamahka, con mayor población de hablantes, están asentados en la reserva Bosawas en los municipios de Bonanza, Siuna y Waspam. Pero, hasta entre los panamahkas, a pesar de ser el grupo mayoritario, que aparentemente conservaba más su lengua, hay comunidades fuera de la reserva que la están perdiendo. Los tuahkas viven en el municipio de Rosita sobre la rivera del Bambina Tungkih, y su lengua está siendo desplazada por el miskitu. Los ulwa viven en el sur de Nicaragua, en la comunidad de Karawala, y su lengua casi no se habla aunque hay esfuerzos para recuperarla.

Los yuskus habitan en la región de Jinotega sobre el río Bocay en dieciséis comunidades; todavía no se sabe si su lengua sobrevivirá, lo cierto es que está en peligro. Los tawahka habitan en Honduras sobre el río Patuca.

Otro de los impulsores en el contacto entre los sumu mayangna y los miskitu fue la llegada de los misioneros moravos, primero en el sur de la región entre los ulwa, y luego, para el año 1906, los misioneros, Newton Wilson y John Fisher, fundaron la congregación de la comunidad de Wasakín (Wilson, 1990: 121).

La influencia de la Iglesia Morava repercutió de manera negativa en la cultura y la lengua sumu-mayangna. Por ejemplo: 
Para el año 1929 llegó a la comunidad de Wasakín el primer pastor moravo de nombre Joe Jiménez, negro de origen colombiano, fue él quien construyó la primera iglesia de Wasakín. Su prédica fue clara y atacó a los sukias porque dijo que eran obra de Satanás, que irían al infierno. El pastor no sólo predicó sino que exigió a los sukias a que abandonaran su fuerza.

Kalu era el sukia de Wasakín, Kalu hizo mucha resistencia. El pueblo de Wasakín lo tenía como la única esperanza. Pero fue obligado a rendirse por la iglesia Morava. Kalu aceptó entonces bautizarse por lo que se cambio de nombre, entonces se llamó Camilo Sailas. Camilo murió en el año 1938. Narrado por Ronas Dolores (citado en Rizo, 2003: 156).

Desde que se fundó la congregación en Wasakín, los pastores moravos fueron en su mayoría de la etnia miskitu o creol. El segundo pastor fue Arnold Steven de origen miskitu de la comunidad de Haulover, este estuvo durante cinco años, luego llego el pastor Robert Care, este era creole de Bluefields, estuvo pastoreando solamente por un año ya que este fue expulsado por violar las normas de la comunidad (Dolores e Ismael, 2004:3).

Después llegó otro pastor de nombre Maibeth Ignacio, miskitu originario de Barata. Posteriormente asumió la dirección de la misión morava el primer pastor sumu tuahka, Miligan Bendlis, originario de Wasakín. Este fue sancionado por la iglesia porque lo acusaron de practicar brujería, es decir él curaba a la gente con hierbas y aceite de coco, aunque nunca aceptó que sus prácticas eran brujería, ya que decía que Dios le había concedido el poder de curar y ayudar a las personas, y era por eso que no cobraba a las personas que curaba. (ibíd.).

Sobre la lengua, el hecho de que se decidiera usar la lengua miskitu para la evangelización tuvo efectos que todavía perduran. Al imponerse la lengua miskitu en un contexto tan importante, se reforzó el poder y el prestigio de aquella lengua, y se disminuyó la prestancia del sumu.

En años más recientes, las relaciones interétnicas en la zona minera entre sumumayangna tuahka y miskitu se han intensificado en diversas formas. En los años 1940-1950, llegan las Compañías Mineras el Rosario Mine Company y la Neptuno Mine Company, que atraen a grandes migraciones de miskitu provenientes del río Coco, Prinzapolka y las zonas del litoral costero que llegaban a Rosita, Bonanza y La Luz (Siuna) para buscar empleos.

Entonces, la relación entre el sumu y el miskitu se establece en primera instancia como una relación de dependencia comercial, es decir basada en una economía de mercado donde los sumus eran los que abastecían con productos, bananos, tubérculos, 
carne, pijibay, pescado y los miskitu quienes tenían el dinero por ser asalariados de la empresa minera.

En la década 1950-1960, en el puente del río Bambana, existía un mercado donde los indígenas tenían plaza para poder vender sus productos y esto los comunitarios lo recuerdan como los mejores momentos que han pasado en la vida económica. Expresan que esta plaza fue promovida por los gringos que administraban la empresa minera, ya que ellos también llegaban a comprar naranjas, mandarinas, cocos y banano [Entrevista MAS Melado, Wasakín, 2006].

Tal a como refiere Houwald (1980):

En los últimos años, los miskitus procedentes de las zonas costeras del Atlántico se han extendido sobre todo subiendo a lo largo de los grandes ríos hasta meterse entre los sumus. Por otro lado, los colonos mestizos se les han aproximado hasta desalojarlos de las tierras cada vez más de sus territorios tradicionales, situación que data desde los años 1894 impulsado por el Estado nicaragüense con la llamada reincorporación de la costa Atlántica, o de la reserva Mosquita (Von Houwald 2001: 5).

Es importante mencionar que estas relaciones estaban marcadas por conflictos y enfrentamientos por una discriminación por parte de los miskitu hacia los sumu.

\section{Consecuencias sociolingüísticas}

Según el trasfondo histórico, algunos de los estudios etnográficos se refieren a la existencia de diez subgrupos de la etnia sumu mayangna, que Smutko denominó "la gran familia sumu". Sin embargo, como consecuencia de la fusión de grupos se provocó la disminución y la extinción de algunos de estos grupos como los prinzu del río Prinzapolka, que cambiaron su lengua y posteriormente fueron considerados como miskitus (Smutko, 1996). Es importante resaltar que este proceso de fusión no solamente se dio entre sumus y miskitus, sino también dentro de la familia sumu. Estos movimientos llevaron a una disminución en el número de lenguas o variantes del sumu.

El dominio de los miskitus, primero a raíz del poder que llegaron a tener por su alianza con los ingleses, y con la adquisición de armas de fuego, el efecto fue que el miskitu se estableciera como una lengua franca entre los grupos indígenas que pagaban tributo al rey mosco. Lo que propició que los sumu aprendieran la lengua miskitu.

La iglesia Morava, en este contexto también ha desempeñado predominancia en el desplazamiento de la lengua sumu. El hecho de usarla como medio para cristianizar a los sumu mayangnas tuvo fuerte impacto por el hecho de que la Biblia la tradujeron 
en lengua miskitu los Moravos. Esto, y el hecho de usar la lengua en las ceremonias religiosas marcaba categorías de valor diferenciado: el miskitu era una lengua importante y escrita, el sumu 'solamente' hablada.

Las relaciones de contacto entre los sumu mayangnas y los miskitus en las minas, crearon situaciones que delimitaban el uso de la lengua sumu tuahka en el caso de la comunidad de Wasakín. Para muchos tuahkas cuando viajaban fuera de la comunidad era vergonzoso hablar su lengua, porque los identificaba como sumu, lo cual ha constituido pretexto de burla por parte de los miskitu.

Más tarde, la presencia de compañías extranjeras en la región también fue una de las causas de reducción de espacios para la lengua sumu, a raíz del intercambio comercial y las dependencias económicas antes referidas. En conjunto, estos procesos crearon una relación de diglosia. El sumu quedó subordinado al miskitu, limitando su uso a contextos de menos prestigio, con lo cual surge una diglosia de fronteras muy rígidas.

\section{Matrimonios mixtos entre mayangna y miskitu y uso de lenguas en el hogar}

Se ubicó un total de 29 parejas mixtas casadas entre tuahka y miskitu, sean hombres miskitus y mujeres tuahkas y viceversa y 6 familias miskitus. Algunas de estas parejas tienen más de 30-40 años de convivir. En muchos casos estas relaciones tienen que ver con la actitud o habilidad de los miskitu, originarios de río Coco, que llegaron a la comunidad para trabajar la tierra y lograron producir grandes cantidades para que no les hiciera falta la comida en el hogar; tener en abundancia las cosechas. Se percibía, pues, una diferencia socioeconómica entre los miskitus y sumus: prevalecía una superioridad del miskitu sobre el sumu, éstos que solamente producían para el consumo y no para la comercialización.

Esta habilidad del hombre miskitu del río Coco, motivó en algunas mujeres mayangnas, el querer casarse con un miskitu en el supuesto de mejorar su posición socioeconómica. Una de las señoras tuahka a quien se entrevistó, recuerda que el hombre miskitu era más laborioso en la agricultura, trabajaba todo el día, cuando cosechaba, era en abundancia; luego, comercializaba el producto y obtenía buenas ganancias que le permitían tener una vida mejor en comparación a las otras familias.

Además, los hombres miskitu tenían trabajos en las minas, obtenían dinero, eran asalariados (MAS Entrevista con Maidela Penn, Wasakín, 2006). En cambio los sumus tuahka hacían sus 'chambas' trasladando maquinarias en los raudales, eran cayuqueros, palanqueros y lo que les pagaban era una miseria en comparación con lo que ganaba el hombre miskitu.

Dentro de las relaciones matrimoniales mixtas sumu-mayangna con miskitu, difícilmente la lengua franca es el sumu tuahka, por el hecho que no existe interés 
por parte del miskitu para aprenderlo. Algunas parejas mixtas hacen uso de ambas lenguas, pero estos casos confirman la excepción.

\section{Movimientos inter-comunales}

Dados los resultados, es evidente que la movilización de los comunitarios de Wasakín en su mayoría se produce hacia comunidades miskitu al ser estas mayorías; por lo tanto, hablar el miskitu es obligatorio para todos los tipos de transacciones y los requerimientos para sobrevivir en esa cultura. En razón que esos contactos se remontan desde hace muchos años, la manera de manifestarse es libre, espontánea.

\section{Uso de las lenguas en los eventos entre comunidades miskitu y mayangna}

En Wasakín se desarrollan diversos eventos de carácter religioso y deportivo donde se reúnen miskitus con sumu-mayangna, panamahka y tuahka, por ejemplo en los encuentros deportivos, partidos de béisbol o fútbol, entre comunidades miskitas de Prinzapolka y los tuahka, así como con las comunidades miskitas que vienen de río Bambana, Ibu, Isnawas, Prinzubila. También estos eventos se dan con grupos de jóvenes mestizos que llegan de Rosita a Wasakín durante los fines de semana. Aquí los jóvenes sumu-mayangnas, tienen cierto dominio del español, lo que posibilita el intercambio de comunicación entre los tuahkas de Wasakín y los mestizos.

Durante el desarrollo de estos eventos, que suelen alternar entre diferentes comunidades, el uso de la lengua varía según las relaciones. A veces se dan situaciones muy contrarias, por ejemplo cuando una conferencia Morava se da en una comunidad panamahka. En estos casos, los panamahka hacen todo en su lengua, cantando, predicando la Biblia, etc., porque ellos difícilmente optan por usar el miskitu como lengua franca. Por lo tanto los tuahka se ven obligados a hacer uso de su lengua tuahka, ya que las dos variantes son mutuamente inteligibles.

Los jóvenes tuahka que no hablan su lengua, ni tampoco el panamahka, muchas veces en estos eventos cantan en miskitu. Los grupos de mayangnas que son bilingües o multilingües se ríen y se burlan en forma de rechazo. En la mayoría de los casos, estos jóvenes son excluidos del grupo mayangna, rechazados por no hablar la lengua mayangna (tuahka), sino el miskitu. Así se produce una situación de discriminación interna dentro de los sumu mayangnas, como una manera de tratar de imponer la variedad panamahka como la mejor.

\section{Los fenómenos de diglosia/poliglosia}

En el marco teórico se apuntalaba la existencia de una situación de diglosia o poliglosia, cuando en una comunidad se habla más de una lengua, estableciéndose una jerarquía que tiene sus raíces en las desigualdades políticas y socioeconómicas entre etnias. En 
tales situaciones cada lengua tiene su espacio y están distribuidas de manera complementaria, donde se usa una lengua no se habla la otra. Por lo tanto es obligatorio hablar una u otra lengua según el contexto comunicativo.

Actualmente se puede afirmar que aún quedan restos de diglosia en la comunidad de Wasakín y el centro urbano de Rosita, donde el español continúa siendo la lengua que se utiliza para todas las gestiones en las instituciones administrativas, por ser la lengua oficial de Nicaragua. A pesar de que se estableció la Ley de Lenguas (Ley 162, 1993), que decreta el uso de las lenguas indígenas en otros ámbitos. Últimamente, la implementación de políticas lingüísticas conforme a esta ley hace que el uso de las lenguas indígenas sea más flexible dentro de las instituciones. También es importante reconocer el avance en cuanto al uso de las lenguas indígenas y étnicas en la educación. Pero la ley no se puede cumplir plenamente hasta que haya mayangna-hablantes calificados para ocupar puestos institucionales.

Hoy en día, entre la mayoría de los jóvenes sumu tuahka de Wasakín, la conversación normalmente se desarrolla en miskitu. Muy pocos se comunican en la lengua tuahka. Cuando un mayangna, hablante del panamahka les conversa a los jóvenes que no hablan en sumu tuahka, estos suelen contestarle en miskitu manteniendo la comunicación en las dos lenguas, entendiéndose bien.

En esta situación los hablantes multilingües utilizan los cambios de código como un recurso extra, para indicar actitudes, cambios de tono en la conversación, como es el caso de aquellos mayangnas panamahkas que son multilingües funcionales, que hacen uso de diversos códigos según sus necesidades e intenciones.

Recientemente se ha generado una relación de subordinación entre las variantes tuahka y el panamahka del sumu (ver gráfica No. 1), para lo cual a manera de hipótesis se plantea tres posibles causas. A raíz de la Revolución Popular Sandinista, en el marco de la Cruzada de Alfabetización en Lenguas, el panamahka tomó auge sobre el tuahka, con la conformación de un equipo técnico de sólo panamahkas.

Esta situación se afianzó con la creación del Programa de Educación Intercultural Bilingüe Mayangna cuyos equipos técnicos fueron integrados sólo por panamahkas, y finalmente con el uso escrito de la variedad panamahka en documentos científicos, educativos, político-económicos y culturales, por ejemplo en el contexto del proceso de la Autonomía. 


\section{Cambio de la lengua mayangna en los Jóvenes tuahka de la comunidad de Wasakín}

Las comunidades tienen un uso restringido según el ámbito en que interactúan; los jóvenes naturalmente se comunican en tuahka, o en lengua miskitu cuando no se tiene un buen dominio de su lengua.

Este fenómeno tiene algunas explicaciones que contradicen los principios del aprendizaje de una lengua, en el caso del tuahka, porque la mayoría de los jóvenes nacen y crecen hablando el miskitu, porque esa es la lengua de comunicación o lengua franca en el hogar, en la comunidad e inclusive en la escuela, y de pronto a medida que estos van creciendo y llegan a la edad juvenil, empiezan a aprender la lengua del grupo étnico como es el caso del tuahka.

Entre los entrevistados, hay 4 estudiantes que han manifestado que desde pequeños sus madres y padres les decían que hablaran solamente en la lengua tuahka, el argumento es que no eran miskitu para hablar miskitu en la casa; sin embargo, uno de los entrevistados manifestaba: "Recuerdo que cuando era pequeño mi papá y mamá nos decían que tenemos que hablar en nuestra propia lengua, pero dentro de la casa ellos siempre nos hablaban en miskitu".

Las comunidades tienen un uso restringido según el ámbito en que interactúan; los jóvenes naturalmente se comunican en tuahka o en lengua miskitu, cuando no se tiene un buen dominio de su lengua.

Entre los entrevistados, hay 4 estudiantes que han manifestado que desde pequeños en sus hogares les decían que hablaran sólo en la lengua tuahka, el argumento es que no eran miskitu para hablar miskitu; sin embargo, uno de los entrevistado manifestaba: "Recuerdo que cuando era pequeño mi papá y mamá nos decían que tenemos que hablar en nuestra propia lengua, pero dentro de la casa ellos siempre nos hablaban en miskitu".

\section{Percepción de las madres y padres de familia, en cuanto a la conservación de la lengua}

Una posible interpretación de los hechos sociolingüísticos que se observó viene de lo que Le Page (1993) llama el "mito genético", o sea de la supuesta relación entre lengua y origen de las naciones. En muchas familias las madres y padres no se preocupan por transmitir la lengua a sus hijas e hijos, porque dicen que una vez crezcan y se hagan jóvenes la hablarán por el mero hecho que llevan la sangre tuahka (Entrevista MAS, Zacarías Angulo, Dibahil, 2006). 
Existe otra creencia relacionada, en las madres de familia tuahkas y panamahkas, donde relacionan la identidad de los hijos con la maternidad, el embarazo. Durante las conversaciones con las madres de familia, frecuentemente manifestaban que si un niño nace del vientre de la madre, este deberá identificarse con el grupo étnico de la madre ya sea sumu, miskitu o mestizo (Asamblea comunitaria. Dibahil, MAS. 2006).

\section{El rol de las madres y padres de familia, en la conservación de la lengua}

Quizás por razones relacionadas a este 'mito', las madres y los padres de familia de la comunidad donde se realizó esta investigación no han demostrado actitudes de querer asumir la responsabilidad de iniciar este proceso de transmisión de la lengua de una generación a otra.

Cuando se les preguntó a algunas madres y padres de familia ¿Quién puede ayudar a resolver el problema de la pérdida de la lengua?, manifestaban de que el problema en el rescate de la lengua sólo lo pueden hacer los que tienen cierto nivel de escolaridad. Consideran que por el hecho de no tener un nivel académico, no pueden hacer nada por el rescate de la lengua tuahka.

Se percibe que muchos conciben su rol como algo parecido al que desempeñan los docentes, y que será difícil que ellos, como madres y padres de familia, puedan ser responsables en el rescate de la lengua.

Es preciso analizar cómo las madres y padres de familia que están inmersos en una situación lingüística compleja, puedan enfrentar y superar estos obstáculos para el rescate de la lengua.

\section{El papel de la escuela en la revitalización de la lengua tuahka}

La escuela es uno de los espacios idóneos donde se debería apoyar el rescate y la enseñanza del tuahka. El Programa de Educación Bilingüe Intercultural (PEBI), manifiesta el respeto a las diferentes culturas en el paradigma de la interculturalidad, planteándose la enseñanza y escritura en las lenguas de los pueblos. Empero, se presentan situaciones contrarias a lo que se espera de una escuela bilingüe en la comunidad.

Los docentes son en su mayoría tuahka, solamente hay un panamahka. Sin embargo, durante el desarrollo de los contenidos de clase el plan de estudio del PEBI, solamente establece que se utilice el tuahka durante un período de noventa minutos. Después de este momento se ha observado que todo transcurre en la lengua miskitu, los docentes entre ellos sólo platican en miskitu, la niñez juega utilizando la lengua miskitu durante todo el día. 
Otro fenómeno por el cual se agudiza el problema de rescatar la lengua sumu tuahka, son los materiales de enseñanza. Todos están elaborados en la variante lingüística panamahka. En el marco del proyecto de Fortalecimiento de la Educación Intercultural Bilingüe en la Costa Caribe, FOREIBCA, un proyecto con fondos del gobierno de Finlandia, inició la elaboración de textos en la variante tuahka para los primeros grados; no obstante, el Ministerio de Educación en Rosita no ha aceptado el uso y validación de estos textos (Asambleas comunitarias, MAS y BRITAC, 2006).

Un contexto multilingüe, el caso de Wasakín y otras comunidades tuahka, donde los esfuerzos de la escuela y la familia están desligados, presenta problemas realmente difíciles para el diseño de un programa bilingüe. El planteamiento actual del PEBI va dirigido hacia la enseñanza de dos idiomas: el sumu L1 y el español, L2. En el caso de comunidades mono-étnicas todo funciona sin problemas. Pero en comunidades como Wasakín, es necesario proponer otro plan de estudios. Por ejemplo, una posible solución sería enseñar el tuahka como segunda lengua. Este tipo de solución se está adoptando en las comunidades ulwa, garífuna y rama, que tienen problemas parecidos (Freeland 2004).

\section{Conclusiones}

\section{Evaluación de franqueza, flexibilidad, explicación, proceso de la investigación, comunicabilidad}

Durante el proceso de la investigación se generó confianza por el hecho de haber seleccionado la comunidad sumu-mayangna tuahka de Wasakín, por muchas razones. Primero porque se han establecido ciertas relaciones de amistad y trabajo con mucha gente de la comunidad; por el conocimiento con propiedad de la lengua sumu-mayangna; el factor tiempo para visitar la comunidad, apoyar y participar con los jóvenes en encuentros deportivos. Esto representó una garantía para obtener la información.

El trabajo de campo fue una gran experiencia en el habérselas con otras realidades culturales y lingüísticas en la comunidad. Por ejemplo, a pesar de que el tema era de importancia para los entrevistados, ellos tenían cierta resistencia en abrirse y contar lo que pasaba, aún a saber que esta investigación concernía al trabajo institucional en cuanto al rescate de la lengua en peligro de extinción.

Así se pudo constatar, que aunque el investigador pertenece a la misma cultura de los investigados, la confianza puede constituirse en una trampa. Por ejemplo, con respecto a la percepción de que todos hablábamos el sumu, se pudo apreciar que cuando se conversaba con las personas de temas que no venían al caso, de cualquier otra cosa, ellas y ellos platicaban muy contentos, hasta hacían bromas y se provocaba a risa. Pero, cuando se abordaba la temática de la investigación, la gente empezaba a 
cuestionar por qué el investigador siendo un panamahka, hablante de otra variante, se interesase por la pérdida de la variante tuahka, porque según sus fuentes los panamahkas fueron los causantes de que el tuahka se perdiera, a consecuencia de imponerles la variante panamahka a través de la escuela, les cerraba la oportunidad a los tuahkas.

Aunque no había razón para sentirse aludido, se evitó imponer algún punto de vista o perspectiva, aclarando oportunamente la razón de la investigación y en referencia al investigador se aclaró que este era del pueblo sumu-mayangna y no tuahka.

También se identificó que el 'hablar la misma lengua' no era tan sencillo como alguien podría imaginar. Cuando se formularon las preguntas de los instrumentos a utilizar, se hizo en español. Sin embargo, cuando se traducían las preguntas al sumu, se traducía a la variante panamahka. Esto hasta cierto momento complicaba un poco el proceso, por el hecho que en la lengua sumu-mayangna no existen ciertos términos técnicos como en el español, y mucho menos en la variante tuahka porque casi no se usa en la escritura de documentos y otros trabajos. Es más, aunque el investigador hablaba el sumu, algunos entendían poco panamahka. Esto obligó a hacer uso de las formas de comunicación no verbal, de los gestos, movimientos corporales para llegar a la comprensión de las preguntas. Es por eso que en algunos casos se repetía hasta dos veces la pregunta.

Al interactuar con las personas entrevistadas al final entendían más de la problemática, fue una situación de aprendizaje mutuo. Eso satisfizo ambas partes, se estaba en el desvelamiento de la verdad en relación a la pérdida de la lengua y la contribución generada hacia la comunidad sumu-mayangna.

\section{Lista de Referencias}

Alzate Álvarez, Dolores María (2001). Informe de la Segunda Salida de Campo. Programa de Reforzamiento de las autoridades comunitarias en la Costa Atlántica de Nicaragua. Municipio de Bonanza y comunidades Mayangnas, RAAN. Managua, Nicaragua.

Bronislaw Malinowski (1884-1942). citado por Álvarez Dolores Ma. \& Grumberg, Jorge (2004). Propedéutico MAS. URACCAN Recinto Universitario de Bilwi R.A.A.N.

Bell, Charles Napier (1862). Citado en Von Houwald, Götz (1984) (2003 [1990]) Mayangna: Apuntes sobre la historia de los indígenas sumu en Centroamérica. Contribuciones a la etnología centroamericana. Managua: Fundación Vida. (Traducción del la edición en alemán, Museo de Etnología de Hamburgo, Vol XIX, 1990.

Conzemius (1932) citado en Von Houwald, Götz (1984) (2003 [1990]) Mayangna: Apuntes sobre la historia de los indígenas sumu en Centroamérica. Contribuciones 
a la etnología centroamericana. Managua: Fundación Vida. (Traducción del la edición en alemán, Museo de Etnología de Hamburgo, Vol XIX, 1990.

Erantz, Martín \& Frank, Eloy (comp.; 2001) Historia oral del pueblo mayangna. FOREIBCA/IPILC/URACCAN. Managua.

Ferguson, C. (1959). "Diglossia”. Word 15, 325-40.

Freeland, Jane (2003). Materiales de Apoyo para la Formación Docente en Educación Intercultural. Tomo 2: Lengua. Managua: IPILC-URACCAN/ Ibis-Kepa-Terra Nuova.

Gómez Sáurez, Agueda (2003). Movilización Política Indígena en las Selvas Latinoamericanas. Los tawahka de la Mosquitia Centroamericana. Editorial Plaza y Valdés S.A. de C.V. México.

Green, Thomas (1996) Perspectivas demográficas e históricas del idioma y el pueblo ulwa, Wani 20: 22-37.

Gurdián, Galio (2001). Mito y memoria en la construcción de la fisonomía de la comunidad de Alamikangban. Disertación Doctoral sin publicar, Universidad de Tejas, Austin.

Hurtado de Mendoza, Luis (2000) Identidad cultural mayangna en Nicaragua. Managua: Sociedad \& Ambiente. Wani 30: 60-66.

Jamieson, Mark (2001). Miskito, sumo y tungla: Variación lingüística e identidad étnica, Wani 27, pp 6-12.

Lehmann (1910) citado en Von Houwald, Götz (1984).

Modood, Tariq (200o). Anti-essentialism, multiculturalism, and the 'recognition' of religious groups [Anti-esencialismo, multiculturalismo, y el 'reconocimiento' de los grupos religiosos], en Kymlicka, Will y Norman Wayne (comp) Citizenship in Diverse Societies [La ciudadanía en las sociedades (étnicamente) diversas] Oxford: Oxford University Press, 2000, pp. 175-195. [Primera sección sobre anti-esencialismo traducida al español por Jane Freeland].

Smutko, Gregorio (1996).La Presencia Capuchina entre los Miskitos 1915-1995. Cartago: Imprenta A.G. Covao.

Wilson F. John (1990). Obra Morava en Nicaragua, Trasfondo y Breve Historia. Editorial UNION CARDOZA y CIA LTDA. Managua - Nicaragua. 